\title{
Comparative studies on the implantation of nano platinum black and pure platinum electrodes in the rabbit orbicularis oculi muscle
}

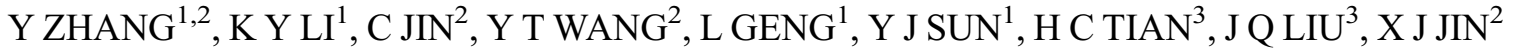 \\ ${ }^{1}$ Department of Otorhinolaryngology Head and Neck Surgery, Shanghai First People's Hospital, Shanghai Jiao \\ Tong University School of Medicine, ${ }^{2}$ Department of Otorhinolaryngology, Shanghai Renji Hospital, Shanghai \\ Jiao Tong University School of Medicine, and ${ }^{3}$ National Key Laboratory of Science and Technology on Nano/ \\ Micro Fabrication Technology, Shanghai Jiao Tong University, PR China
}

\begin{abstract}
Objective: To study the interactive influence of implanted nano platinum black electrodes (as compared with pure platinum electrodes) on rabbit orbicularis oculi muscle morphology and function.

Methods: The influence of the two types of electrode on the orbicularis oculi muscle was monitored in a rabbit model of facial paralysis. Biological electric current and exciting current were administered to biological tissue, and morphological and functional changes were identified. Changes in orbicularis oculi muscle contraction, electrode configuration and performance associated with long-term electrical stimulation were observed over 28 days of implantation.

Results: The nano platinum black electrode was superior to the pure platinum electrode in the following aspects: morphology and functionality, electrical excitation function of the orbicularis oculi muscle (as assessed by electromyography), muscle contraction function and biological tissue changes. Furthermore, the nano platinum black electrode features had good stability.

Conclusion: Microelectrode surface modification with nano platinum black can effectively increase the microelectrode surface area and improve electrode performance, and is associated with good tissue compatibility.
\end{abstract}

Key words: Parylene; Platinum Black; Implantable Electrodes; Microelectrodes

\section{Introduction}

Facial paralysis often causes muscle tone loss, limited facial expression and eye closure dysfunction as a result of facial nerve injury. The most serious consequence for patients with facial paralysis is blindness due to functional deficits in eyelid closing. ${ }^{1}$ The reasons for facial nerve injury usually include palsy, trauma, infection and inflammation. ${ }^{2}$

There are currently no effective treatments for longterm peripheral facial paralysis; hence, we proposed and investigated a new treatment method. Specifically, we established an artificial facial nerve reflex to restore the denervation of facial features. We used an electronic chip to replace the control centre of the facial nerve. The size of the electronic chip is similar to that used in artificial cochleas; it can be implanted into the frontal sinus on the injured side or other areas. The excitation potential information of the contralateral facial nerve is accepted by the microelectrode, and is analysed and determined by the central electronic chips. The current output is used (in a corresponding mode) to directly stimulate the facial area of the injured side with multiple electrodes. The bilateral facial muscles are prompted to contract synchronously, with the same pattern. This enables the maintenance of facial expression structure symmetry, and the correction of deficits such as eyelid closure weakness, commissure distortion and nasolabial groove shallowing. In addition, disuse facial muscle atrophy can be prevented by stimulating a slight contraction of the facial muscles on the injured side with a random distribution of electric current.

In our study on implants, we employed $20-\mu \mathrm{m}$ diameter platinum wire as the microelectrode material. This wire is very thin and has high electrical impedance. We therefore coated the wire with nano platinum black, which significantly decreases the electrical impedance. ${ }^{3}$ However, the interaction between such electrodes and organisms needs further clarification. The use of platinum wire as an implanted electrode material 
has a long history, and there are many reports in the literature. ${ }^{4}$ The effects of platinum wire on the morphology and function of biological tissue are different after they are coated with nano platinum black. Biological tissues and body fluids also respond to electrode electrolysis, corrosion and electrical characteristics in different ways. ${ }^{5,6}$ These differences must be controlled within a certain range to guarantee proper function of the implanted materials in the facial nerve system and prevent harm to biological tissue. Implant studies are critical for clarifying and addressing these effects and differences.

\section{Materials and methods}

\section{Animals and equipment}

Twenty New Zealand white rabbits (male to female ratio of $1: 1$ ) were used in the study (permission numbers: $\operatorname{SCXK}(\mathrm{SH}) 2012-0007$ and $\operatorname{SYXK}(\mathrm{SH})$ 2009-0086).

Equipment included a scanning electron microscope (Ultra 55; Zeiss, Oberkochen, Germany), a 4-channel electromyography (EMG) evoked potentiometer (NTS-2000; NCC, Shanghai, China), a manual slicer (RM2235; Leica, Nussloch, Germany), a microscope (BX51; Olympus, Tokyo, Japan), and electroplating and testing instruments (Chenhua 660c; $\mathrm{CH}$ Instruments, Shanghai, China).

\section{Electrode preparation}

The fabricated, flexible nano platinum black coated electrodes and traditional pure platinum electrodes were prepared using an implantable microelectromechanical technique. The myoelectricity-gathering microelectrode was implanted in the contralateral side to acquire the signal generated by the muscles, and the stimulating microelectrode was implanted in the affected side. A biocompatible polymer parylene film, made with a plasma-enhanced chemical vapour deposition system, was used to cover the electrode and platinum wire $(0.02 \mathrm{~mm}$ in diameter), acting as a flexible insulator. ${ }^{7}$ Photolithography and oxygen deep reactive ion-etching techniques were applied to the exposed electrode.

\section{Animal model preparation}

All procedures used for this animal model of unilateral peripheral facial paralysis were conducted using sterile techniques according to the protocols approved by the Ethics Committee for Animal Research, Shanghai First People's Hospital affiliated to Shanghai Jiaotong University, PR China. No deaths occurred during anaesthesia to implant the electrodes. The rabbits recovered strength 1 hour after anaesthesia, at which time they ate and drank normally. No apparent dysaesthesia was observed.

Adult New Zealand white rabbits were used for the chronic electrode implantation experiments. After they were anaesthetised with 1 per cent pentobarbital sodium $(3 \mathrm{ml} / \mathrm{kg})$ through the marginal ear vein, the rabbits were immobilised on a stereotaxic frame. First, the junction of the left auricle cartilage and bone border was identified. Then, the skin on the surface of the masseter muscle and the subcutaneous tissue was cut to expose the facial nerve branches. A facial nerve monitoring probe (NTS-2000; NCC) was used to stimulate the nerve branch, and masseter and jaw twitches were observed. These nerve branches extend towards the proximal end, and join superior and posterior nerves to converge into the stylomastoid foramen. Eyelid, ear, cheek and neck muscle twitches were observed while the facial nerve stem segment exiting the stylomastoid foramen was stimulated by the probe (Figures 1 and 2). A partial facial nerve stem segment at the stylomastoid foramen was removed, and the distal and proximal ends were turned to prevent recanalisation. The severed distal and proximal ends of the nerve were stimulated by the facial nerve monitoring probe 10 minutes later; disconnection was confirmed if there was no twitching observed at the sides of the ears and eyes, or in the neck muscles.

All incisions were stitched up layer by layer. All stitches were removed after 7 days and the rabbits' facial expressions were observed. Electromyography was performed to examine orbicularis oculi muscle activity on the surgical (left) side to confirm the denervation (Figure 3 ).

\section{Electrode implantation}

The upper and lower eyelid skin was regularly sterilised. A $5 \mathrm{ml}$ guided needle was used to penetrate from the lateral limbus of the eye epidermis to the inside near the inner canthus. The electrode wire was inserted via the guide needle, which was then carefully removed. The electrode head was left in the orbicularis oculi muscle. The platinum-coated part of the nano platinum black electrode was placed within the orbicularis oculi muscle. Both exposed ends of the electrodes were fixed with stitches and glue. Electrodes were

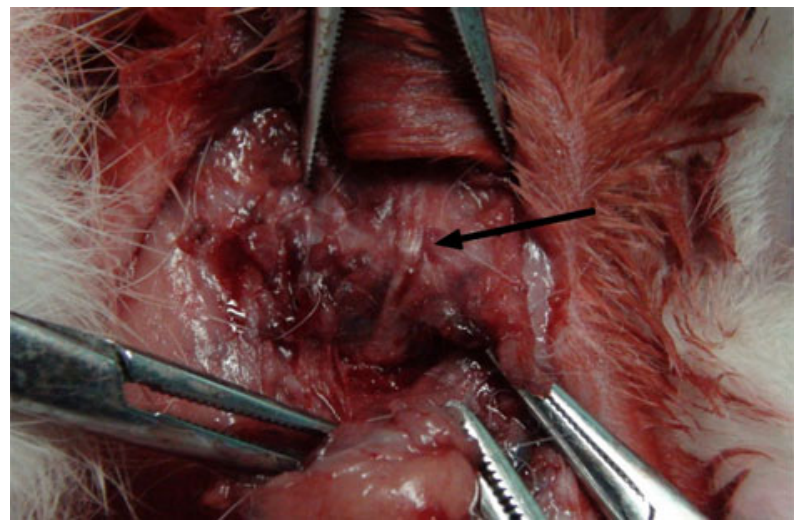

FIG. 1

Rabbit facial nerve anatomy (arrow indicates the stem segment of the facial nerve). 


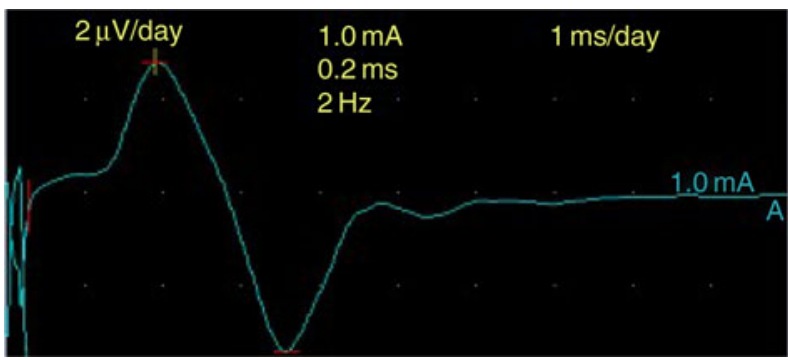

FIG. 2

Electromyography performed during rabbit facial nerve dissection, showing effect of $1.0 \mathrm{~mA}$ stimulation on the stem segment of the facial nerve.

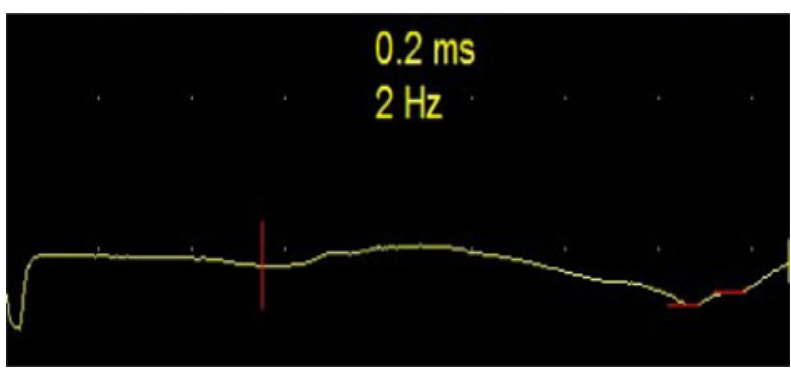

FIG. 3

Electromyography confirmed denervation after complete removal of the stem segment of the facial nerve (stimulation did not elicit waveform).

embedded into the superior and inferior orbicularis oculi using the same method.

\section{Animal groups}

For the experimental group, 10 pairs of number-ranked nano platinum black electrodes were implanted into 10 animals (one electrode on the surgical side and one on the non-surgical side) into the orbicularis oculi muscle under scanning electron microscopy, and electrical characteristics were measured. The nerve on the nonsurgical side was connected to the EMG recording device and muscle activity was measured 4 hours per day for 28 days. These measurements were used to calculate daily average amplitude and to draw measurement curves. The nerve on the surgical side was connected to the electrical stimulator for 1 hour per day (providing continuous stimulation at $1.0 \mathrm{~mA}$ intensity) for 28 days. Changes to the palpebral fissure were evaluated with a video recording, and measurement curves were drawn. After the rabbits had been sacrificed, the microelectrodes were removed. Electron microscope scanning was conducted and the electrical characteristics were measured. The orbicularis oculi muscle was then removed and fixed with formaldehyde. This was followed by haematoxylin and eosin slice staining and light microscopy analysis.

Except for the use of pure platinum electrodes in the control group, the treatment process for this group $(n=10)$ was the same as in the experimental group.

\section{Data collection}

Following electrode implantation, electrical excitation of the orbicularis oculi muscle was determined daily by EMG, and muscle contraction function was ascertained by the palpebral fissure size. Morphological changes were established by biological observations of tissue conducted after the animals had been sacrificed. The method used to induce eye closure is a commonly used technique. In our experiments, filamentous cotton was used to touch the rabbit cornea with continuous stimulation for 10 seconds, and eye closure was measured with EMG (i.e. activity reflecting induced eye closure, as opposed to spontaneous eye closure occurring without external stimulation ${ }^{8}$ ). The average amplitude related to induced eye closure for the two types of electrodes was recorded for 28 days for statistical comparison. Over the same 28-day period, induced minimum palpebral fissure distance with $1 \mathrm{~mA}$ current stimulation and the maximum palpebral fissure distance without stimulation were recorded for the two groups, and the ratio of the two measurements (per cent) (referred to as closed eyes palpebral fissure ratio) was statistically analysed.

\section{Electrode effects on tissue cells}

The rabbits were sacrificed 28 days after implantation in order to observe the effects of electrode insertion on tissue cells. The implanted area of the orbicularis oculi muscle was isolated and fixed with formalin. The tissue sample was then embedded in paraffin, and stained with haematoxylin and eosin. The morphological patterns of the surrounding cells of the electrodeimplanted antrum were observed by light microscopy. The number of eosinophilic and lymphocyte cells was counted under the same high power field $(\times 100)$ with the antrum placed at the centre.

\section{Cell tissue fluid effect on electrode performance}

The morphological patterns of the electrodes were examined with an electron microscope before and after the experiments. The influence of the cell tissue fluid on electrode performance was evaluated by assessing cyclic voltammetry curves and performing electrochemical impedance spectroscopy., 9

\section{Statistical method}

Data analyses were performed using SPSS software, version 18.0 (SPSS, Chicago, Illinois, USA) and the means were compared using paired $t$-tests at a significance level of $p<0.05$.

\section{Results}

\section{Electrode morphology}

Electron microscopy revealed that the surface of the nano platinum black electrode was coarse and had a cauliflower-like appearance before implantation (Figure 4). This surface increased the effective area of the electrode point and reduced the impedance. 


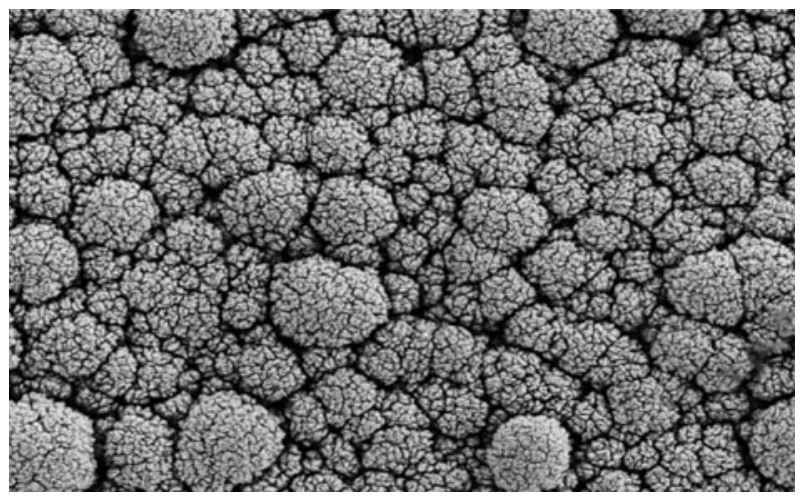

FIG. 4

Electron microscopy image of a nano platinum black electrode before implantation. $(\times 10000)$

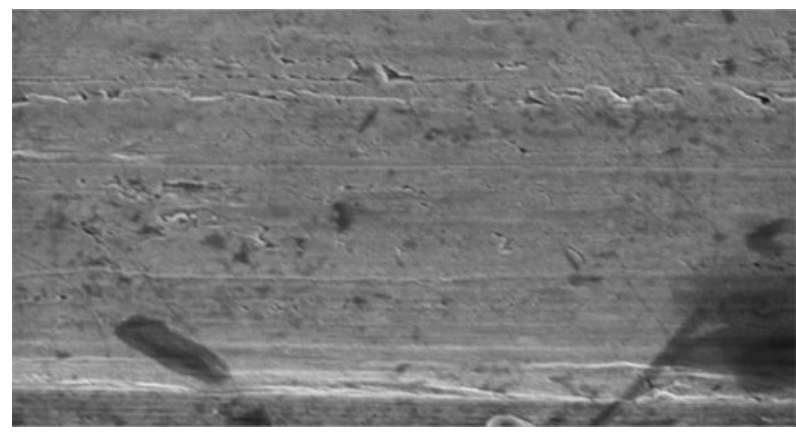

FIG. 5

Electron microscopy image of a pure platinum electrode before implantation. $(\times 10000)$

TABLE I

AVERAGE ELECTRODE IMPEDANCE IN ELECTROCHEMICAL IMPEDANCE SPECTROSCOPY*

\begin{tabular}{lcc}
\hline Electrode & Before implantation & After implantation \\
\hline Nano platinum black & $99.61 \pm 3.82$ & $78.14 \pm 1.88$ \\
Pure platinum & $8165.00 \pm 183.36$ & $388.1 \pm 1.83$ \\
\hline
\end{tabular}

*Impedance curve at $1 \mathrm{kHz}$ frequency (mean \pm standard deviation; $\Omega$ ); 10 animals per (electrode) group.

The surface of the pure platinum electrode before implantation was smooth, so the effective area was smaller and the impedance was large (Figure 5). The average electrode impedances of the nano platinum black and pure platinum electrodes at $1 \mathrm{kHz}$ frequency were $99.61 \Omega$ and $8165 \Omega$, respectively (Table I). There were no obvious morphological changes for either electrode type after implantation (Figures 6 and 7).

\section{Electrode performance}

Average electrode function test parameter findings for the 20 animals are shown in Figures 8-13. The yellow line in the figures represents electrode function before implantation. The black line indicates electrode function after implantation.

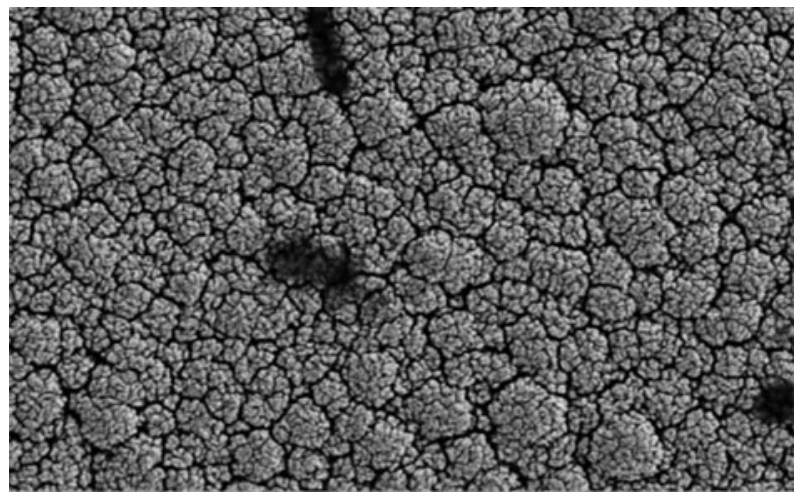

FIG. 6

Electron microscopy image of a nano platinum black electrode after implantation. $(\times 10000)$

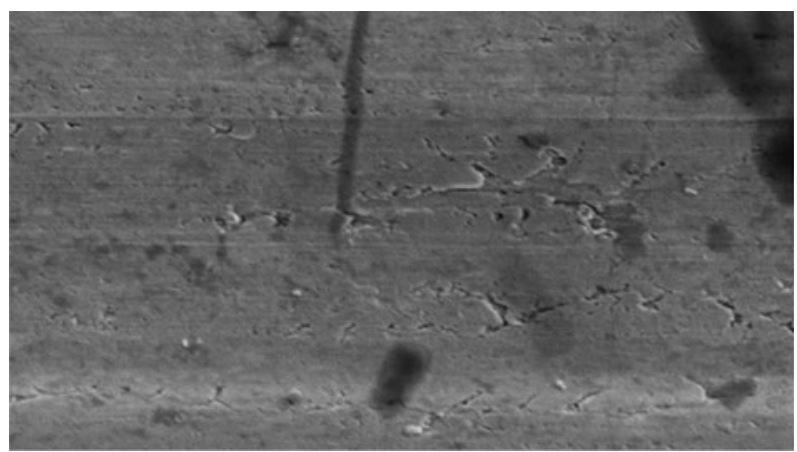

FIG. 7

Electron microscopy image of a pure platinum electrode after implantation. $(\times 10000)$

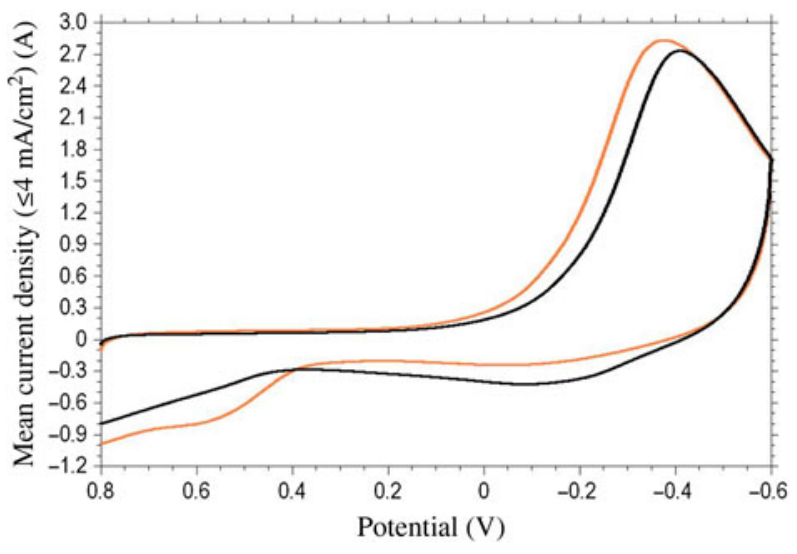

FIG. 8

Cyclic voltammetry curves for the nano platinum black electrode (orange line $=$ before implantation; black line $=$ after removal, following 28 days of implantation).

\section{Electromyography findings}

Signal acquisition electrodes recorded the electrical signals of the orbicularis oculi muscle in vivo. The EMG signal was stable while the rabbit's uninjured side was still and the eye was open, and there was no obvious amplitude. Over time, the EMG signal amplitude was higher for the nano platinum black electrode 


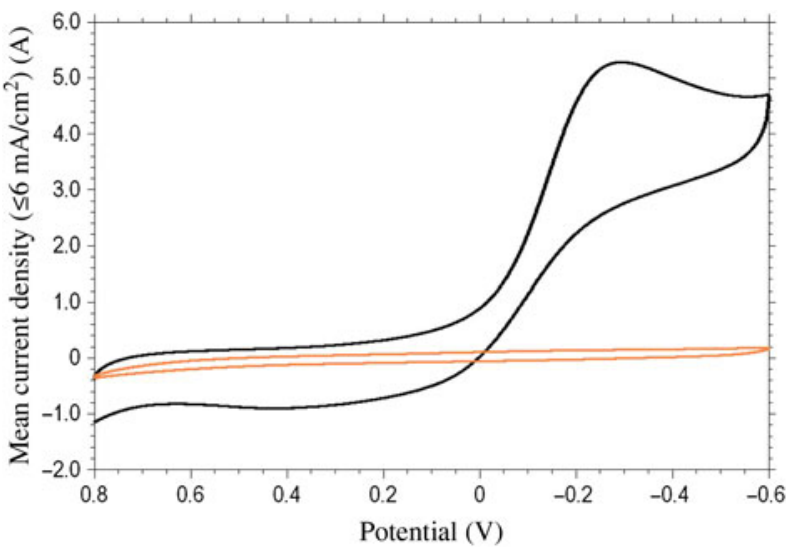

FIG. 9

Cyclic voltammetry curves for the pure platinum electrode (orange line $=$ before implantation; black line $=$ after removal, following 28 days of implantation).

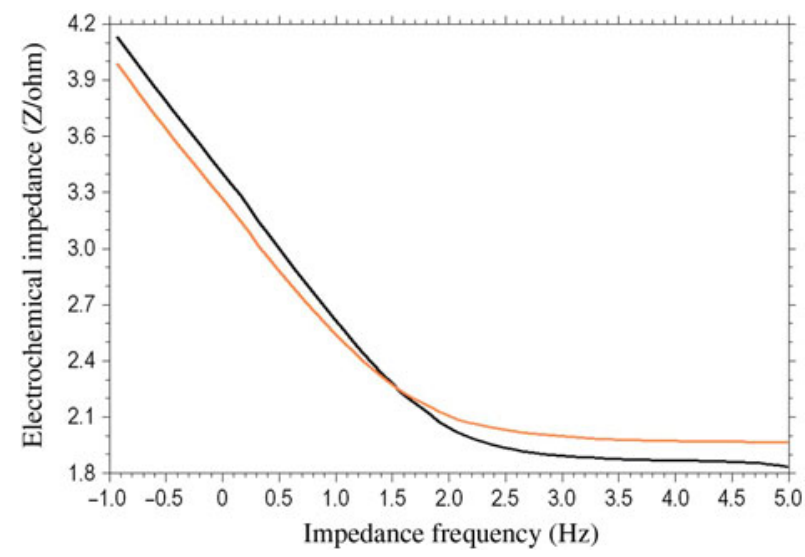

FIG. 10

Impedance curve of electrochemical impedance spectroscopy for the nano platinum black electrode (orange line = before implantation; black line $=$ after removal, following 28 days of implantation).

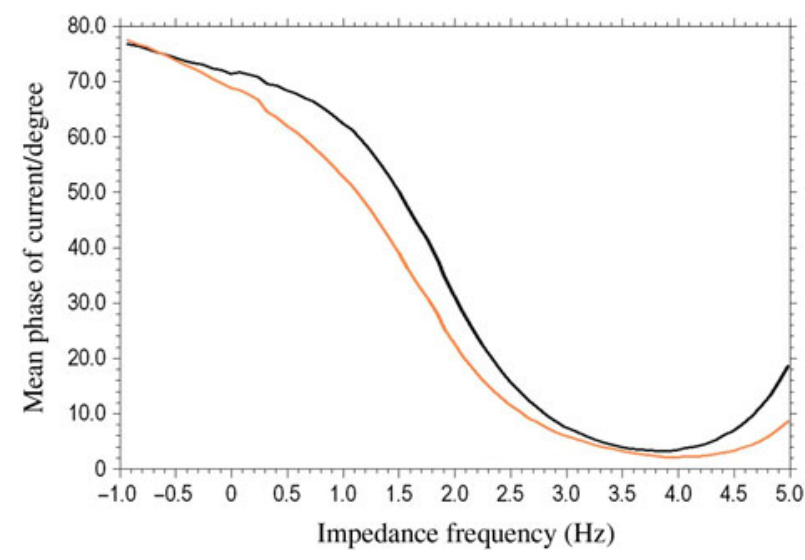

FIG. 11

Phase curve of electrochemical impedance spectroscopy for the nano platinum black electrode (orange line = before implantation; black line $=$ after removal, following 28 days of implantation)

compared with the pure platinum electrode during the orbicularis oculi muscle contractions caused by induced eye closure (Figures 14-17, Table II).

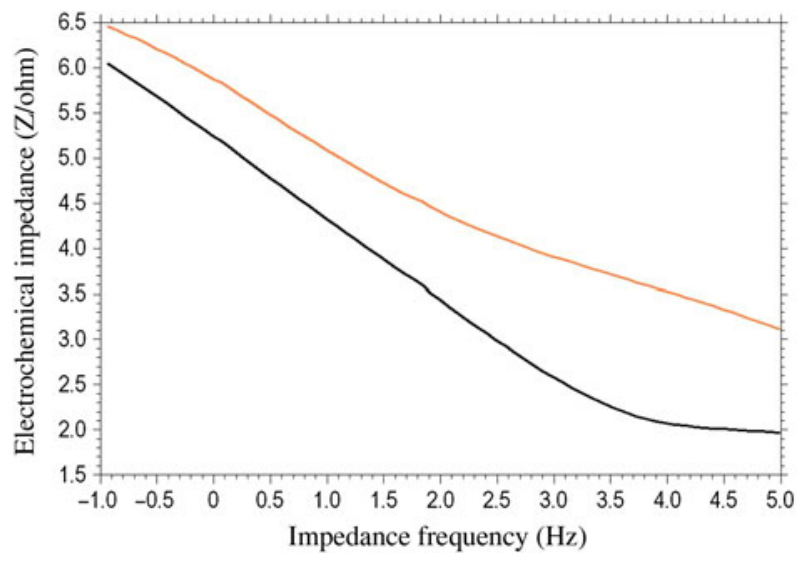

FIG. 12

Impedance curve of electrochemical impedance spectroscopy for the pure platinum electrode (orange line $=$ before implantation; black line $=$ after removal, following 28 days of implantation).

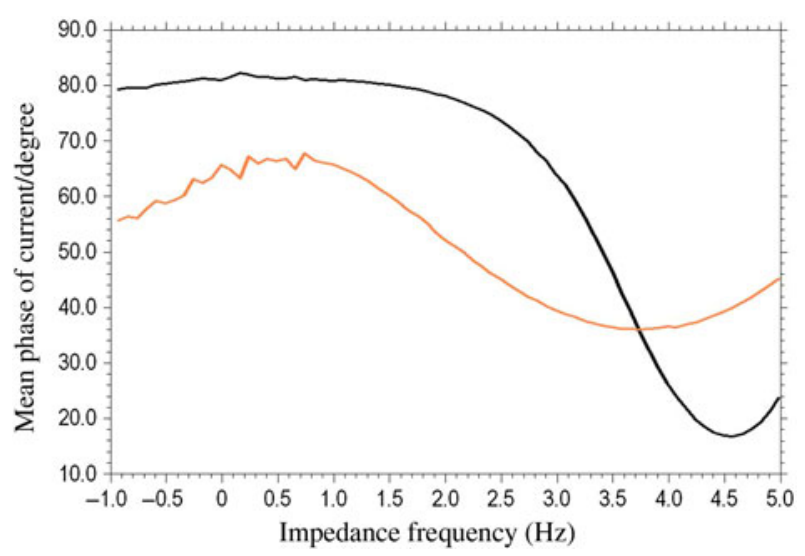

FIG. 13

Phase curve of electrochemical impedance spectroscopy for the pure platinum electrode (orange line = before implantation; black line $=$ after removal, following 28 days of implantation).

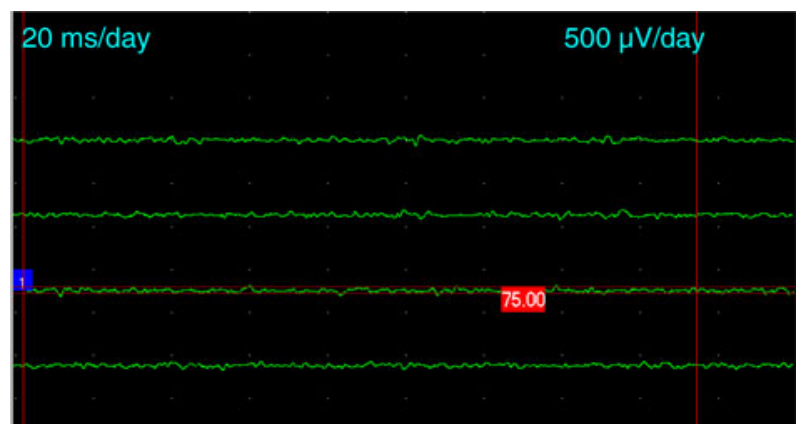

FIG. 14

Pure platinum electrode monitoring: electromyography graph of rabbit orbicularis oculi muscle activity during a static, open eye condition.

There was a significant difference between the two types of electrode in terms of the average EMG amplitude associated with induced eye closure of the contralateral side after 15 days (see Figure 18 and Table II). 


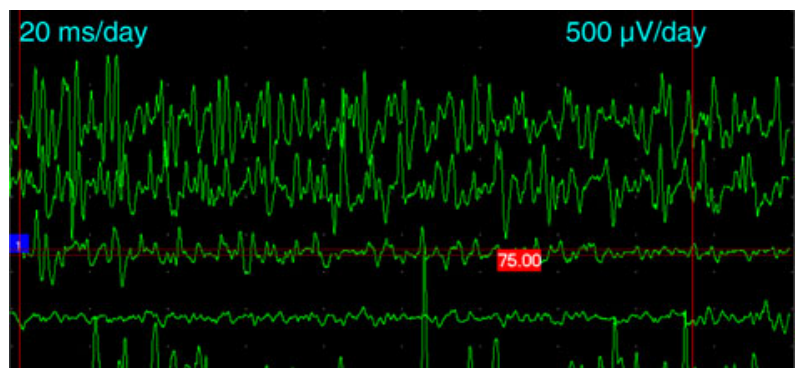

FIG. 15

Pure platinum electrode monitoring: electromyography graph of rabbit orbicularis oculi muscle activity during a continuous, induced eye closure condition.

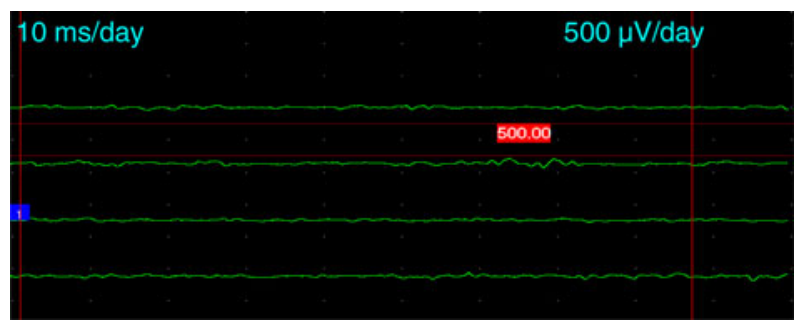

FIG. 16

Nano platinum black electrode monitoring: electromyography graph of rabbit orbicularis oculi muscle activity during a static, open eye condition.

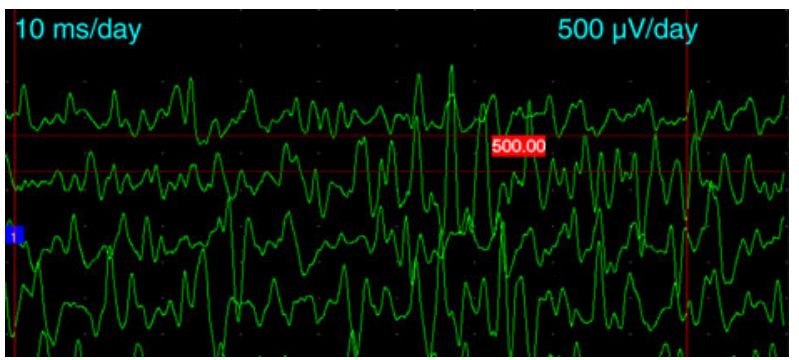

FIG. 17

Nano platinum black electrode monitoring: electromyography graph of rabbit orbicularis oculi muscle activity during a continuous, induced eye closure condition.

\section{Closed eyes palpebral fissure ratio}

The induced closed eyes palpebral fissure ratio was used to determine electrical stimulation efficiency. This is the ratio of the minimum closed eyes palpebral fissure induced by $1 \mathrm{~mA}$ current stimulation and the maximum palpebral fissure without stimulation (per cent). The results obtained over 28 days are shown in Figure 19 and Table III.

The impedance of the nano platinum black electrode was less than that measured for the pure platinum electrode, and its conduction was more sensitive throughout the experimental period. The closed eyes palpebral fissure ratio was significantly lower for the nano platinum black electrode treatment compared with the pure platinum electrode treatment.

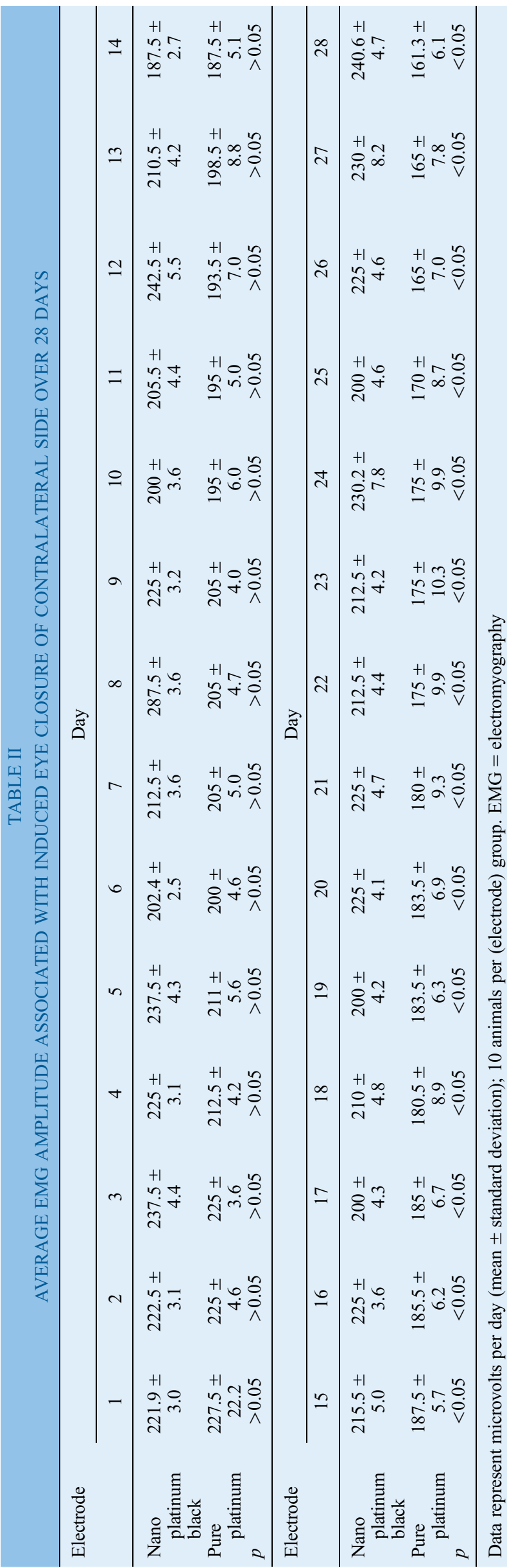




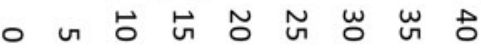

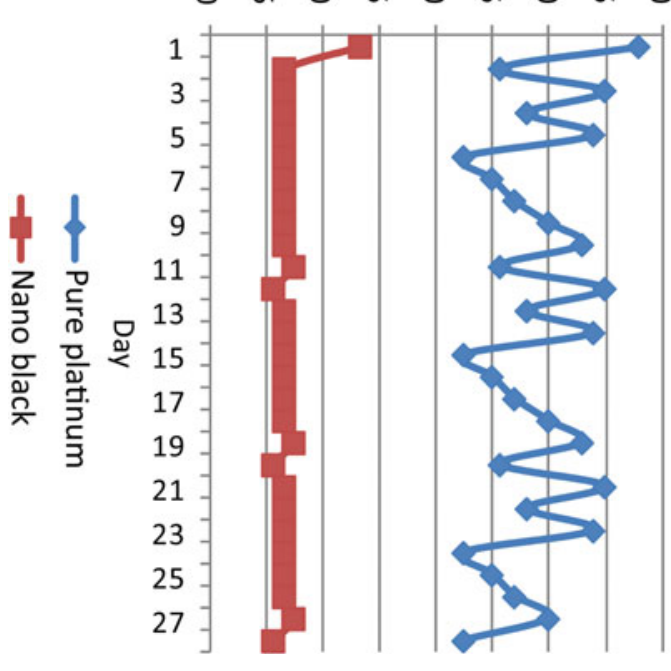

Closed eyes palpebral fissure ratio (\%)

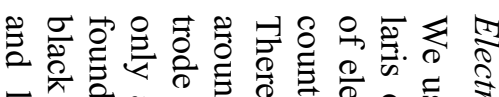

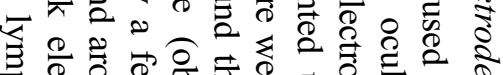
㐘号方 क $\rightarrow$.

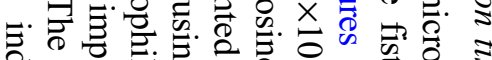
त्ञ

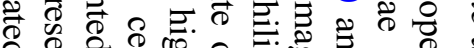
居

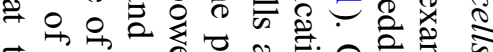

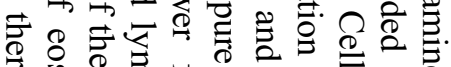

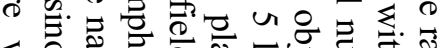

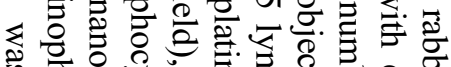
든

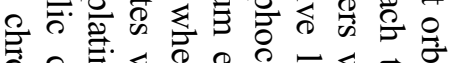
总.

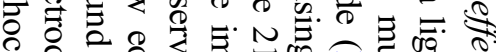

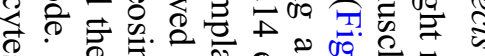

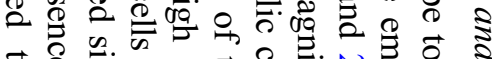
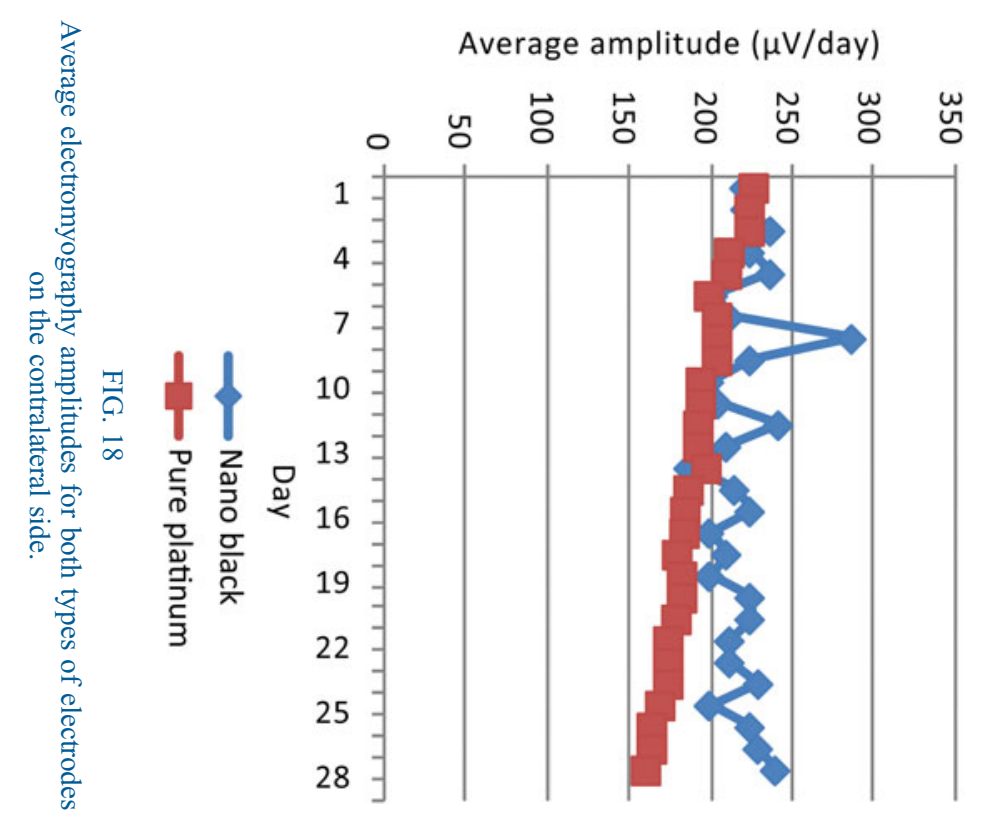

Average amplitude ( $\mu \mathrm{V} /$ day)

TABLE III

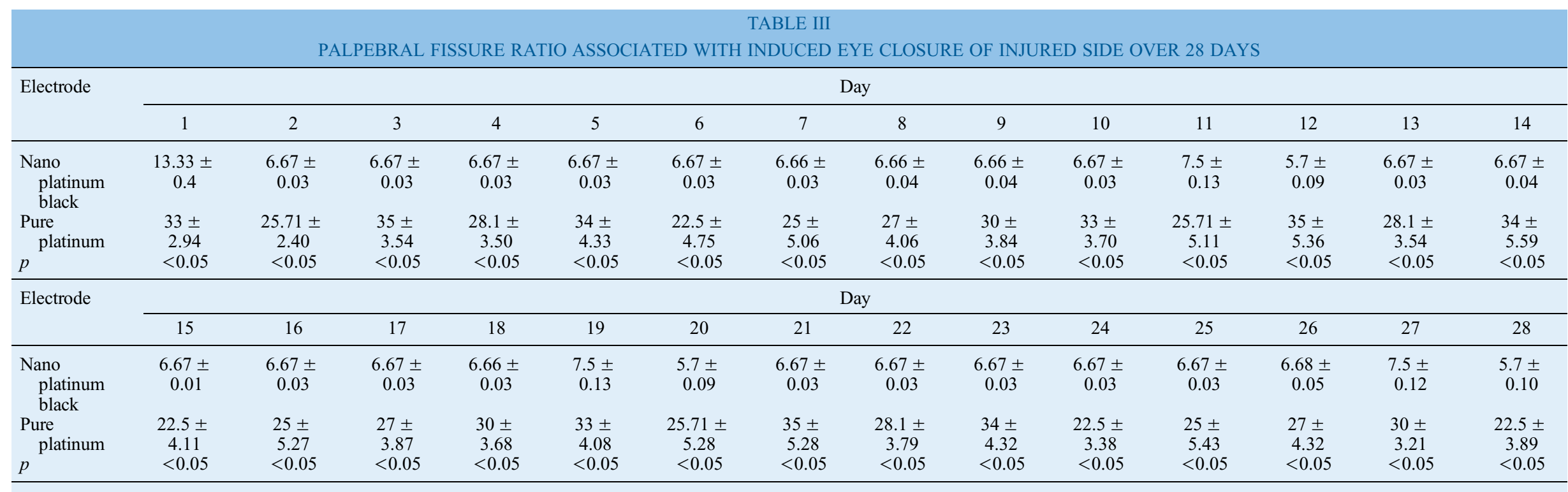




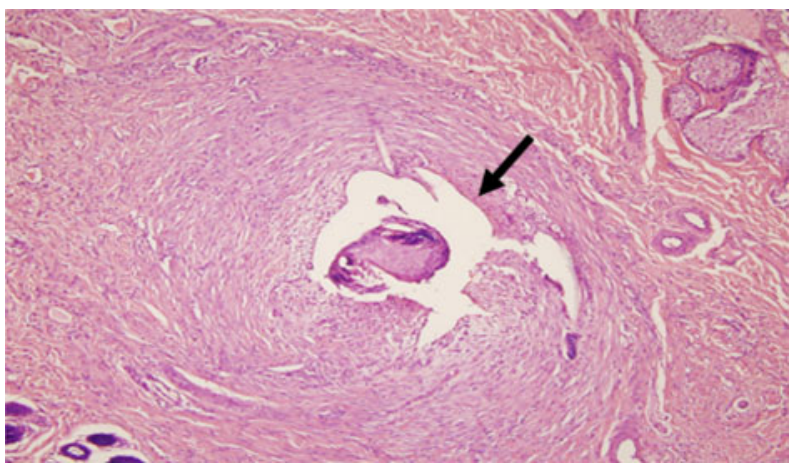

FIG. 20

Light microscopy image of rabbit orbicularis oculi muscle fistula embedded with a nano platinum black electrode (arrow indicates fistula margin, showing few eosinophilic cells and lymphocytes). $(\mathrm{H} \& \mathrm{E} ; \times 100)$

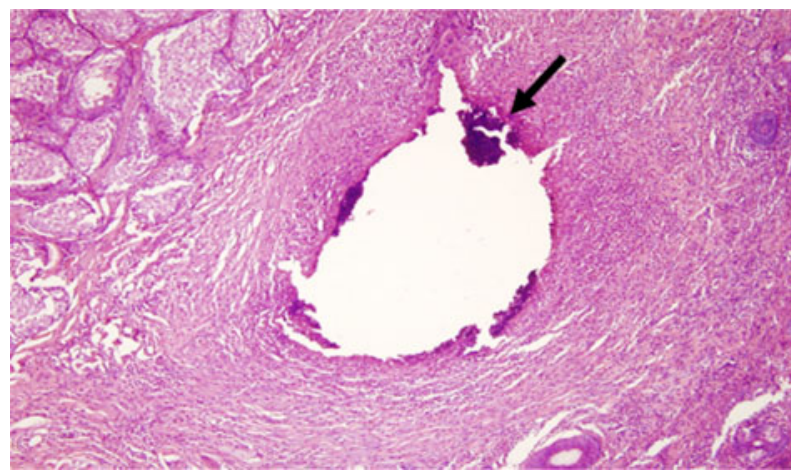

FIG. 2

Light microscopy image of rabbit orbicularis oculi muscle fistula embedded with pure platinum electrode (arrow indicates hyperchromatic, eosinophilic cells and lymphocytes, observed around the edge of the fistula). $(\mathrm{H} \& \mathrm{E} ; \times 100)$

inflammation around the implanted site of the pure platinum electrode and that the nano platinum black electrode had better tissue compatibility. ${ }^{11}$ There was no significant difference between the recording electrodes and the stimulating electrode in terms of the effects on tissue or cells.

The numbers of inflammatory cells surrounding the antrum (i.e. the sum of eosinophilic cells and lymphocytes), present after removal of the two electrode types, were examined and compared by light microscopy under $\times 100$ magnification $(n=20)$; the results are shown in Table IV. Within the same high power fields, we found a significant difference in the number of inflammatory cells between the areas around the two types of electrode on the surgical side. However, no significant difference in inflammatory cell numbers was found between the areas on the opposite side.

\section{Discussion}

The applications of implantable functional electrical stimulators are gradually being increased. The diameter of the microelectrode is limited by the small size of

\begin{tabular}{lccc}
\multicolumn{4}{c}{ TABLE IV } \\
\multicolumn{4}{c}{$\begin{array}{c}\text { INFLAMMATORY CELL INFILTRATION LEVELS } \\
\text { AROUND ELECTRODES }\end{array}$} \\
\hline Electrode & Recording side & $\begin{array}{c}\text { Stimulating } \\
\text { side }\end{array}$ & $p$ \\
& \multicolumn{4}{c}{$\begin{array}{c}1.51 \pm 0.09 \\
\text { Nano platinum } \\
\text { black }(n)\end{array}$} & $>0.05$ \\
$\begin{array}{l}\text { Pure platinum }(n) \\
p\end{array}$ & $\begin{array}{c}202.81 \pm 0.05 \\
<0.001\end{array}$ & $\begin{array}{c}205.31 \pm 4.43 \\
<0.001\end{array}$ & $>0.05$ \\
\hline
\end{tabular}

Data represent averages (mean \pm standard deviation); 10 animals per (electrode) group. Columns reflect a comparison of the two electrode types for the same side; rows represent a comparison with the same electrode for the two sides.

muscle cells, and the microelectrodes implanted in muscle tissue can only be manufactured in a size ranging from around a few micrometres to tens of micrometres. Narrowing the diameter of the microelectrode can increase its impedance dramatically and can reach the megohm level. The microelectrode impedance becomes the main source of coupled thermal noise and environmental noise compared to the resistance with other components. ${ }^{12}$

Platinum is commonly applied as a sensitive material for conductance electrodes. In order to reduce the polarisation effect, we added a black coating to the platinum surface. This increases the electrode surface area, which decreases current density and the polarisation effect, and the capacitance interference can decrease dramatically as a consequence. The coarse surface of the black particles dramatically increases the effective surface area, thereby greatly reducing microelectrode impedance. ${ }^{13}$ In our experiment, after 28 days of electrode implantation in the rabbit orbicularis oculi muscle in vivo, there were no obvious changes found for the two types of electrodes before and after implantation, as observed using electron microscopy (Figures 4-7). Even after several weeks, biological tissues and fluids had not yet caused significant changes in electrode configuration.

After electrode implantation, the biological tissue and fluids will eventually affect electrode electrolysis, corrosion and electrical characteristics to a certain level. Cyclic voltammetry curves and electrochemical impedance spectroscopy are commonly employed electrode performance parameters. ${ }^{14,15}$ Cyclic voltammetry curves are determined by controlling electrode potential at different rates with a triangular waveform, with one-time or repeated scanning. The potential range makes the electrode alternate between different reduction and oxidation reactions, and the current-voltage potential curve can then be recorded. According to the curve shape, we can determine: the level of electrode reaction and whether the reaction is reversible, the intermediates of phase formation, interfacial adsorption, the possibility of new phase formation, the properties of coupled chemical reactions, and so on. The curve shape is commonly used to measure electrode reaction parameters, determine control steps and 
the reaction mechanism, and to monitor the potential scanning range for possible reactions and their properties. For a new electrochemical system, the preferred research method is cyclic voltammetry 'electrochemical spectroscopy'. ${ }^{16}$

In cyclic voltammetry tests, charge storage capacity represents the degree of electrode impairment. In our experiment, the results showed that the charge storage capacity of the nano platinum black electrodes decreased very little after implantation, indicating that the nano platinum black coating caused little damage (Figure 8). The pure platinum electrode charge storage capacity reduced significantly after implantation, indicating that the effective area of the electrode increased and caused more damage (Figure 9). It may be that the parylene film on the insulating layer of the pure platinum electrode and the exposed electrode point contributed to this effect. The nano-modified coating and exposed electrode surface guaranteed consistency between the electrode's insulating layer and the protective membrane on the exposed electrode. So, in the process of long-term preparations, the insulating layer of the pure platinum electrode and the prick stitch can easily be aggravated and cause greater damage.

Electrochemical impedance spectroscopy is a technique used to apply a different frequency wave of small amplitude alternating current (AC) potential on the electrochemical system. We then measure the AC potential and current signal ratio (this ratio is the system impedance) with the sine wave frequency $(\omega)$ changes or the impedance phase angle $(\Phi)$ with $\omega$ changes. ${ }^{17}$ The technique can be extended to analyse electrode kinetics, double layers and diffusion, electrode materials, and solid electrolytes, for conducting polymers and corrosion protection mechanisms for example. Electrochemical impedance spectroscopy impedance curves and phase curves after implantation were very small for the nano platinum black electrode, indicating stability of the electrode material (Figures 10 and 11). For the pure platinum electrode, the phase curve was larger because of decreases in electrode impedance, increases in valid electrode area and damage to the insulating layer, all of which suggest poor material stability (Figures 12 and 13).

The electrode index of $1 \mathrm{kHz}$ impedance was an important reference index. This frequency is associated with neural signal recording and is related to electric energy loss in the process of nerve stimulation. ${ }^{18}$ The $1 \mathrm{kHz}$ frequency electrode impedance curves (Table I) showed that the impedance values of the nano platinum black electrode were less than those of the pure platinum electrode both before and after implantation. The impedance of the nano platinum black electrode decreased slightly after implantation, indicating that there was no obvious damage to the nano platinum black electrode. Pure platinum electrode impedance decreased significantly after implantation, probably due to the lack of the nanoparticle layer protection. We found that wear, corrosion resistance and stability were superior for the nano platinum black electrode compared with the pure platinum electrode.

The morphology and function assessments showed that the nano platinum black electrodes remained stable after implantation. The electrode shape and electrical characteristics did not change significantly. Although the pure platinum electrode did not show obvious morphological damage, its function decreased significantly. These findings indicate that the nano platinum black materials are more stable than the pure platinum materials.

The results for the contralateral orbicularis oculi muscle electrical signals, for the nano electrode (experimental) group and the pure platinum electrode (control) group, showed that the two electrode types led to different orbicularis oculi muscle electrical excitation functions under different muscle-electrical conditions during the 28 implantation days (Figures 14-17). Analysis of the average contralateral amplitudes for the two electrodes types, from days 1 to 28 of the implantation period, revealed a statistically significant change in the amplitude of the pure platinum electrode only (Table II and Figure 18). The difference between electrode types was statistically significant from the 15th implantation day onwards. This finding indicates that the functional impairment of the pure platinum electrodes gradually increased over time as a result of a certain amount of accumulation. In the first 14 days, the fluid stimulation and the friction on the electrode from biological activities caused irreversible functional damage to the pure platinum electrodes (without a nano coating), and this led to a significant difference in average amplitude for the recording electrode. The protection on the electrode point and the insulating layer on the nano electrodes withstood the friction from biological activity and body fluids, indicating that the nano platinum black electrode was functionally stable.

- Nano platinum black electrodes are superior to pure platinum electrodes in terms of morphology and functionality

- The nano platinum black electrode features have good stability

- Microelectrode surface modification with nano platinum black can effectively increase the microelectrode surface area, improve electrode performance and yield good tissue compatibility

The effects of the current-stimulating electrode on the denervated rabbit orbicularis oculi muscle were compared over 28 days. This revealed significant differences over time between the two types of electrode in terms of the ratio of the minimum palpebral fissure 
distance induced by $1 \mathrm{~mA}$ current stimulation and the maximum palpebral fissure distance when there was no stimulation on the surgical side (Figure 19, Table III). This finding is likely to be a result of the low electrode impedance and good conductive sensitivity of the nano platinum black electrode, resulting in a low minimum and maximum palpebral fissure ratio.

Implanted electrodes are made of biocompatible materials, but long-term implantation can cause different degrees of tissue inflammatory reactions. ${ }^{19}$ Fibrous tissue cysts surrounding the electrode significantly increase electrode impedances, and cause muscle tissue and electrode separation. This increases the stimulus threshold and energy consumption of the implanted device, which dramatically affects the implanted device operating results. ${ }^{20}$ Therefore, electrode surface bioactivity modification methods have attracted considerable attention. $^{21,22}$ This study examined images of fistulae surrounding the nano platinum black and pure platinum electrodes after 28 days of implantation in the rabbit orbicularis oculi muscle. The inflammatory cells were counted under light microscopy at $\times 100$ magnification and the data were statistically analysed (Figures 20 and 21, Table IV). The results revealed visible rings of eosinophilic cells and some lymphocytes surrounding pure platinum electrode fistulae, but there were only a few eosinophilic cells and lymphocytes surrounding the nano platinum black electrode. The number of inflammatory cells was significantly different between the two types of electrodes (Table IV). However, there was no significant difference between the recording and stimulating sides (Table IV). The results suggest that the difference in inflammatory cell appearance is related to the type of electrode material rather than the presence of different cells between the recording and stimulating sides. The presence of eosinophilic cells and lymphocytes indicated chronic inflammation surrounding the pure platinum electrode and strong repellency towards the foreign body. Conversely, there were no obvious inflammation reactions surrounding the nano platinum black electrodes. It is clear that the nano platinum black electrodes have considerable tissue compatibility advantages compared with the pure platinum electrodes.

\section{Conclusion}

This study describes a modified implanted electrode for facial paralysis treatment. In order to improve electrode performance, we used the biocompatible polymer film parylene, which is made using a chemical vapour deposition technique, as a flexible insulator to cover the electrode and wire. Photolithography and oxygen deep reactive ion etching were subsequently used to expose the electrode, and this was followed by nano platinum coating to cover the exposed surface in order to reduce electrode impedance.

Scanning electron microscopy showed that the nano black modified layer of the platinum surface had a good morphological surface, with dense and uniform distribution. The morphological and functional comparison of the nano black platinum and the pure platinum electrodes, over 28 days of implantation, indicated that the nano black platinum electrode had no obvious influence on biological tissue, and biological tissues and fluids did not appear to influence electrolysis, corrosion or the electrical characteristics of the nano black platinum electrode. Conversely, the pure platinum electrode significantly affected biological tissue morphology and function, and the function of the pure platinum electrode clearly declined (without obvious morphological change) over 28 days of implantation.

Nano platinum black electrodes can be safely implanted in rabbit orbicularis oculi muscles, and used to record and stimulate physiological activities. With the nano platinum black electrode, stimulation can be applied in high values. Furthermore, the electrode is very sensitive, offers real-time detection of physiological activities and has a good histocompatibility. These advantages of the nano platinum black electrode can ensure that implanted artificial nerve systems work properly, without damaging biological tissue. Therefore, the nano platinum black electrodes have a broad application for peripheral facial paralysis research and treatment.

\section{Acknowledgements}

This work was funded by the National Natural Science Foundation of China (grant number 81070779), and the Science and Technology Commission of Shanghai Key Research Projects. The authors are thankful for financial support received from the Science and Technology Commission of Shanghai (grant number 11441900102). The authors are also thankful for the partial financial support received from the National Natural Science Foundation of China (grant numbers 51035005 and 61076107).

\section{References}

1 Ljostad U, Okstad S, Topstad T, Mygland A, Monstad P. Acute peripheral facial palsy in adults. $J$ Neurol 2005;252:672-6

2 Lu Y, Wang D, Li T, Zhao X, Cao Y, Yang H et al. Poly(vinyl alcohol)/poly(acrylic acid) hydrogel coatings for improving electrode-neural tissue interface. Biomaterials 2009;30:4143-51

3 Manal K, Gonzalez RV, Lloyd DG, Buchanan TS. A real-time EMG-driven virtual arm. Comput Biol Med 2002;32:25-36

4 Wessberg J, Stambaugh CR, Kralik JD, Beck PD, Laubach M, Chapin JK et al. Real-time prediction of hand trajectory by ensembles of cortical neurons in primates. Nature 2000;408: 361-5

5 Serruya MD, Hatsopoulos NG, Paninski L, Fellows MR, Donoghue JP. Instant neural control of a movement signal. Nature 2002;416:141-2

6 Somia NN, Zonnevijlle ED, Stremel RW, Maldonado C, Gossman MD, Barker JH. Multi-channel orbicularis oculi stimulation to restore eye-blink function in facial paralysis. Microsurgery 2001;21:264-70

7 Otto RA. Restoration of function in the paralyzed rabbit orbicularis oculi muscle by direct functional electrical stimulation. Laryngoscope 1997;107:101-11

8 Zealear DL, Rodriguez RJ, Kenny T, Billante MJ, Cho Y, Billante CR et al. Electrical stimulation of a denervated muscle promotes selective reinnervation by native over foreign motoneurons. J Neurophysiol 2002;87:2195-9 
9 Targan RS, Alon G, Kay SL. Effect of long-term electrical stimulation on motor recovery and improvement of clinical residuals in patients with unresolved facial nerve palsy. Otolaryngol Head Neck Surg 2000;122:246-52

10 Smit JE, Hanekom T, Hanekom JJ. Estimation of stimulus attenuation in cochlear implants. J Neurosci Methods 2009; 180:363-73

11 Hoag H. Remote control. Nature 2003;423:796-8

12 Hochberg LR, Serruya MD, Friehs GM, Mukand JA, Saleh M, Caplan AH et al. Neuronal ensemble control of prosthetic devices by a human with tetraplegia. Nature 2006;442:164-71

$13 \mathrm{Lu} \mathrm{Y,} \mathrm{Li} \mathrm{T,} \mathrm{Zhao} \mathrm{X,} \mathrm{Li} \mathrm{M,} \mathrm{Cao} \mathrm{Y,} \mathrm{Yang} \mathrm{H}$ et al. Electrodeposited polypyrrole/carbon nanotubes composite films electrodes for neural interfaces. Biomaterials 2010;31: 5169-81

14 Cogan SF. Neural stimulation and recording electrodes. Annu Rev Biomed Eng 2008;10:275-309

15 Schwartz AB. Cortical neural prosthetics. Annu Rev Neurosci 2004;27:487-507

16 Bisquert J. Theory of the impedance of electron diffusion and recombination in a thin layer. J Phys Chem B 2002;106: 325-33

17 Maynard EM. Visual prostheses. Annu Rev Biomed Eng 2001;3: $145-68$

18 Jackson A, Mavoori J, Fetz EE. Long-term motor cortex plasticity induced by an electronic neural implant. Nature 2006;444 $56-60$

19 Rui YF, Liu JQ, Yang B, Li KY, Yang CS. Parylene-based implantable platinum-black coated wire microelectrode for orbicularis oculi muscle electrical stimulation. Biomed Microdevices 2012;14:367-73

20 Peng C, Jin J, Chen GZ. A comparative study on electrochemical co-deposition and capacitance of composite films of conducting polymers and carbon nanotubes. Electrochim Acta 2007;53: 525-37

21 Dongyue X, Keyong L, Jingquan L, Yujuan W, Yuefeng R. Quantitative features of myoelectric signals in the orbicularis oculi muscle during different motion states. Neural Regeneration Research 2010;5:1895-9

22 Yujuan W, Keyong Li, Jingquan L, Dongyue X, Yuefeng R, Chunsheng Y. Artificial facial nerve reflex restores eyelid closure following orbicularis oculi muscle denervation. Neural Regeneration Research 2010;5:1750-5

Address for correspondence:

Dr K Y Li,

Department of Otorhinolaryngology Head and Neck Surgery,

Shanghai First People's Hospital,

Shanghai Jiao Tong University School of Medicine,

Shanghai 200080, PR China

Fax: +8663240825

E-mail: likeyong5@126.com

Dr K Y Li takes responsibility for the integrity of the content of the paper

Competing interests: None declared 